\section{Exergaming: An Effective Way to Maintain Physical and Mental Health at Home during COVID-19 Pandemic}

Sir,

The recent pandemic of severe acute respiratory syndrome coronavirus 2 (SARS-CoV-2), also known as coronavirus disease 2019 (COVID-19), has adversely affected the life of masses all across the world. The ones who got infected by this deadly virus have been quarantined and self-isolated for months. In the best interests of general public, the government of Pakistan implemented the state of lockdown in the country dating back in the month of March, 2020 and it led to a situation where everyone including adults, kids and elderly were home-struck with little healthy recreational physical activities; and the situation is still the same. ${ }^{1}$ The physical and mental health have also been seriously affected among all age groups. Gyms, parks and sports clubs are all closed and people are left with quite limited health promoting alternatives. So, in this scenario, exergaming is an advisable option to maintain and promote physical as well as mental health. It works on the concept of virtual reality where integration takes place between exercise and physical activity through digital games. Exergaming encompasses various kinds of physical activities including walking, running, cycling, swimming, stair climbing, rowing, baseball, tennis, Frisbee, boxing, golf, stretching and strengthening exercises, dancing and yoga, etc. ${ }^{2}$ The gaming devices have been found to improve the physical and mental health and are very safe to use at home. ${ }^{2,3}$ It has also been observed that the exergaming reduces the anxiety and stress occurring because of isolation in COVID-19 outbreak. ${ }^{2}$

Moreover, staying active also improves the immunity, which plays a crucial role in fighting against the microorganism-mediated ailments including COVID-19. Apart from boosting the immune system, physical exercise, which might not prevent the development of this disease, reduces the negative impact that is brought about by stress of living in isolation. ${ }^{4}$ Literature also supports that the physically active patients have shorter recovery period with less severe symptoms. ${ }^{5}$ Physical exercises using exergaming platforms not only improve the fitness in healthy individuals, butalso those suffering from diseases. ${ }^{3}$

Exergaming devices are user-friendly and are equipped with biofeedback system, which enhances the motivational level of the user. They are easily available in all the major cities of Pakistan. The most reliable, locally available brands are
Nintendo and X-Box. Their price varies from as low as PKR 10,000 for a refurbished console to 60,000 for a new one. In order to effectively achieve the benefits of exercising at home, exergaming is a best tool.

\section{CONFLICT OF INTEREST:}

The authors declared no conflict of interest.

\section{AUTHORS' CONTRIBUTION:}

NS: Concept, design and accountable for this work. KK: Drafting, revision and accountable for this work. MMAA: Critical analysis, final approval and accountable for this work.

\section{REFERENCES}

1. Nation T. Pakistan continues to observe partial lockdown across country. Islamabad, Pakistan: Nawaiwaqt group; 2020 [cited 2020 July 8]; Available from: http:// nation.com.pk/26-Mar-2020/partial-lockdown-continuesacross-pakistan.

2. Viana RB, de Lira CAB. Exergames as Coping Strategies for Anxiety Disorders during the COVID-19 Quarantine Period. Games for Health J 2020; 9(3):147-9.

3. Khushnood K, Sultan N, Mehmood R, Qureshi S, Tariq H, Amjad I. Does Wii Fit balance training improve balance and reduce fall risk in diabetic patients as compared to balance training exercises? A randomised control trial. Rawal Med J 2019; 44(1):44-8.

4. Simpson RJ, Katsanis E. The immunological case for staying active during the COVID-19 pandemic. Brain Behav Immun 2020; 87:6-7. doi: 10.1016/j.bbi.2020.04.041.

5. Duggal NA, Niemiro G, Harridge SD, Simpson RJ, Lord JM. Can physical activity ameliorate immunosenescence and thereby reduce age-related multi-morbidity? Nat Rev Immunol 2019; 19(9):563-72. doi: 10.1038/s41577-0190177-9.

Nasir Sultan ${ }^{1}$, Kiran Khushnood ${ }^{2}$ and Malik Muhammad Ali Awan ${ }^{2}$

${ }^{1}$ Department of Rehabilitation Sciences, Shifa Tameer-e-Millat University, Islamabad, Pakistan

${ }^{2}$ Isra Institute of Rehabilitation Sciences, Isra University, Islamabad, Pakistan

Correspondence to: Dr. Malik Muhammad Ali Awan, Isra Institute of Rehabilitation Sciences, Isra University, Islamabad, Pakistan

E-mail: mmaawan@gmail.com

Received: June 20, 2020; Revised: July 09, 2020; Accepted: July 09, 2020

DOI: https://doi.org/10.29271/jcpsp.2020.JCPSPCR.CR143 